\title{
Existence of solutions for fractional $q$-difference equation with mixed nonlinear boundary conditions
}

\author{
Xinhui Li ${ }^{1}$, Zhenlai Han ${ }^{1 *}$, Shurong Sun ${ }^{1}$ and Ping Zhao ${ }^{2}$
}

"Correspondence:

hanzhenlai@163.com

'School of Mathematical Sciences,

University of Jinan, Jinan, Shandong 250022, P.R. China

Full list of author information is available at the end of the article

\begin{abstract}
In this paper, we study the boundary value problem for a class of nonlinear fractional $q$-difference equation with mixed nonlinear conditions involving the fractional $q$-derivative of Riemann-Liouuville type. By means of the Guo-Krasnosel'skii fixed point theorem on cones, some results concerning the existence of solutions are obtained. Finally, examples are presented to illustrate our main results.
\end{abstract}

MSC: 39A13; 34B18; 34A08

Keywords: fractional q-difference equations; mixed nonlinear conditions; fixed point theorem in cones; existence of solutions

\section{Introduction}

Fractional calculus is a generalization of integer order calculus [1,2]. It has been used by many researchers to adequately describe the evolution of a variety of engineering, economical, physical, and biological processes [3]. There are a large number of papers dealing with the continuous fractional calculus. Among all the topics, boundary value problems for fractional differential equations have attracted considerable attention [4-6]. However, the discrete fractional calculus has seen slower progress, it is still a relatively new and emerging area of mathematics. Some efforts have also been made to develop the theory of discrete fractional calculus in various directions. For some recent works, see [7]. Of particular note is that Atici and Sengül have shown the usefulness of fractional difference equations in tumor growth modeling in [8].

The early works about $q$-difference calculus or quantum calculus were first developed by Jackson $[9,10]$, while basic definitions and properties can be found in the monograph by Kac and Cheung [11]. $q$-Difference equations have been widely used in mathematical physical problems, dynamical system and quantum models [12], heat and wave equations [13], and sampling theory of signal analysis [14].

As an important part of discrete mathematics, more recently, some researchers devoted their attention to the study of the fractional $q$-difference calculus, they developed the $q$-analogs of fractional integral and difference operators properties, the $q$-Mittag-Leffler function [15], $q$-Laplace transform, $q$-Taylor's formula, etc. [16]. The origin of the fractional $q$-difference calculus can be traced back to the works in $[17,18]$ by Al-Salam and

(อ2014 Li et al.: licensee Springer. This is an Open Access article distributed under the terms of the Creative Commons Attribution License (http://creativecommons.org/licenses/by/2.0), which permits unrestricted use, distribution, and reproduction in any medium, provided the original work is properly cited. 
Agarwal. A book on this subject by Annaby and Mansour [19] summarizes and organizes much of the $q$-fractional calculus and equations.

As is well known, the aim of finding solutions to boundary value problems is of main importance in various fields of applied mathematics. Recently, there seems to be a new interest in the study of the boundary value problems for fractional $q$-difference equations [20-27].

In 2012, Liang and Zhang [21] studied the three-point boundary problem of fractional $q$-differences,

$$
\begin{aligned}
& \left(D_{q}^{\alpha} u\right)(t)+f(t, u(t))=0, \quad 0<t<1,2<\alpha<3, \\
& u(0)=\left(D_{q} u\right)(0)=0, \quad\left(D_{q} u\right)(1)=\beta\left(D_{q} u\right)(\eta),
\end{aligned}
$$

where $0<\beta \eta^{\alpha-2}<1$. By using a fixed point theorem in partially ordered sets, they got some sufficient conditions for the existence and uniqueness of positive solutions to the above boundary problem.

In 2013, Zhou and Liu [22] studied the existence results for fractional $q$-difference equations with nonlocal $q$-integral boundary conditions,

$$
\begin{aligned}
& \left(D_{q}^{\alpha} u\right)(t)+f(t, u(t))=0, \quad t \in(0,1), \\
& u(0)=0, \quad u(1)=\mu I_{q}^{\beta} u(\eta)=\mu \int_{0}^{\eta} \frac{(\eta-q s)^{(\beta-1)}}{\Gamma_{q}(\beta)} u(s) d_{q} s,
\end{aligned}
$$

where $\mu>0$ is a parameter, $D_{q}^{\alpha}$ is the $q$-derivative of Riemann-Liouville type of order $\alpha$. By using the generalized Banach contraction principle, the monotone iterative method and Krasnoselskii's fixed point theorem, some existence results of positive solutions to the above boundary value problems were enunciated.

In 2013, Goodrich [28] proved that the nonlocal boundary value problem with mixed nonlinear boundary conditions

$$
\begin{aligned}
& -y^{\prime \prime}(t)=f(t, y(t)), \quad 0<t<1, \\
& y(0)=H_{1}(\varphi(y))+\int_{E} H_{2}(s, y(s)) d s, \quad y(1)=0,
\end{aligned}
$$

has at least one positive solution by imposing some relatively mild structural conditions on $f, H_{1}, H_{2}$ and $\varphi$.

To the best of our knowledge, very few authors consider the boundary value problem of fractional $q$-difference equations with mixed nonlinear boundary conditions. Theories and applications seem to be just initiated. This paper will fill up the gap. Here, motivated by [28], we will consider the boundary value problem of the nonlinear fractional $q$-difference equations

$$
\left(D_{q}^{\alpha} u\right)(t)+f(t, u(t))=0, \quad 0<t<1,
$$

subject to the boundary conditions

$$
D_{q}^{i} u(0)=0, \quad i=0,1, \ldots, n-2, \quad D_{q} u(1)=H_{1}(\varphi(u))+\int_{E} H_{2}(s, u(s)) d_{q} s,
$$


where $0<q<1, n-1<\alpha \leq n, n \geq 2$ are integers, $f:[0,1] \times[0, \infty) \rightarrow[0,+\infty)$ is continuous and $\varphi$ is a linear functional, here $E \subseteq(0,1]$ is a closed subinterval, $H_{1}:[0,+\infty) \rightarrow[0,+\infty)$ and $H_{2}:[0,1] \times[0,+\infty) \rightarrow[0,+\infty)$ are real-valued, continuous functions. We are interested in the existence of solutions for the boundary value problem (1.1)-(1.2) by utilizing a fixed point theorem on cones.

We should mention that the above boundary conditions are rather general and contain many common cases such as separated boundary conditions, integral boundary conditions, multi-point boundary conditions, etc., by choosing different $H_{1}, H_{2}$, and $\phi$. Our results generalize and improve some results on the existence of solutions for fractional $q$-difference equations. Moreover, problems studied in [20] and [27] can be regarded as our special cases.

The paper is organized as follows. In Section 2, we introduce some definitions of $q$-fractional integral and differential operator together with some basic properties and lemmas to prove our main results. In Section 3, we investigate the existence of solutions for the boundary value problem (1.1)-(1.2) by fixed point theorem on cones. Moreover, some examples are given to illustrate our main results.

\section{Preliminaries}

In the following section, we collect some definitions and lemmas about fractional $q$-integral and fractional $q$-derivative; for an overview of the theory one is referred to $[18,20]$.

Let $q \in(0,1)$ and define

$$
[a]_{q}=\frac{1-q^{a}}{1-q}, \quad a \in \mathbb{R}
$$

The $q$-analog of the power function $(a-b)^{n}$ with $n \in \mathbb{N}_{0}$ is

$$
(a-b)^{0}=1, \quad(a-b)^{n}=\prod_{k=0}^{n-1}\left(a-b q^{k}\right), \quad n \in \mathbb{N}, a, b \in \mathbb{R} .
$$

More generally, if $\alpha \in \mathbb{R}$, then

$$
(a-b)^{(\alpha)}=a^{\alpha} \prod_{n=0}^{\infty} \frac{a-b q^{n}}{a-b q^{\alpha+n}} .
$$

It is easy to see that $[a(t-s)]^{(\alpha)}=a^{\alpha}(t-s)^{(\alpha)}$. Note that, if $b=0$ then $a^{(\alpha)}=a^{\alpha}$.

The $q$-gamma function is defined by

$$
\Gamma_{q}(x)=\frac{(1-q)^{(x-1)}}{(1-q)^{x-1}}, \quad x \in \mathbb{R} \backslash\{0,-1,-2, \ldots\},
$$

and satisfies $\Gamma_{q}(x+1)=[x]_{q} \Gamma_{q}(x)$.

The $q$-derivative of a function $f$ is here defined by

$$
\left(D_{q} f\right)(x)=\frac{f(x)-f(q x)}{(1-q) x}, \quad\left(D_{q} f\right)(0)=\lim _{x \rightarrow 0}\left(D_{q} f\right)(x) \quad \text { for } x \neq 0
$$


and $q$-derivatives of higher order by

$$
\left(D_{q}^{0} f\right)(x)=f(x) \quad \text { and } \quad\left(D_{q}^{n} f\right)(x)=D_{q}\left(D_{q}^{n-1} f\right)(x), \quad n \in \mathbb{N} .
$$

The $q$-integral of a function $f$ defined on the interval $[0, b]$ is given by

$$
\left(I_{q} f\right)(x)=\int_{0}^{x} f(t) d_{q} t=x(1-q) \sum_{n=0}^{\infty} f\left(x q^{n}\right) q^{n}, \quad x \in[0, b] .
$$

From the definition of $q$-integral and the properties of series, we can get the following results on $q$-integral, which are helpful in the proofs of our main results.

\section{Lemma 2.1}

(1) If $f$ and $g$ are $q$-integral on the interval $[a, b], \alpha \in \mathbb{R}, c \in[a, b]$, then

(i) $\int_{a}^{b}(f(t)+g(t)) d_{q} t=\int_{a}^{b} f(t) d_{q} t+\int_{a}^{b} g(t) d_{q} t$;

(ii) $\int_{a}^{b} \alpha f(t) d_{q} t=\alpha \int_{b}^{a} f(t) d_{q} t$;

(iii) $\int_{a}^{b} f(t) d_{q} t=\int_{a}^{c} f(t) d_{q} t+\int_{c}^{b} f(t) d_{q} t$.

(2) If $|f|$ is q-integral on the interval $[0, x]$, then $\left|\int_{0}^{x} f(t) d_{q} t\right| \leq \int_{0}^{x}|f(t)| d_{q} t$.

(3) Iff and $g$ are $q$-integral on the interval $[0, x], f(t) \leq g(t)$ for all $t \in[0, x]$, then $\int_{0}^{x} f(t) d_{q} t \leq \int_{0}^{x} g(t) d_{q} t$.

Basic properties of $q$-integral and $q$-differential operators can be found in the book [11]. We now present out three formulas that will be used later $\left({ }_{i} D_{q}\right.$ denotes the derivative with respect to variable $i$ )

$$
\begin{aligned}
& { }_{t} D_{q}(t-s)^{(\alpha)}=[\alpha]_{q}(t-s)^{(\alpha-1)}, \\
& \left({ }_{x} D_{q} \int_{0}^{x} f(x, t) d_{q} t\right)(x)=\int_{0}^{x}{ }_{x} D_{q} f(x, t) d_{q} t+f(q x, x) .
\end{aligned}
$$

Remark 2.1 We note that if $\alpha>0$ and $a \leq b \leq t$, then $(t-a)^{(\alpha)} \geq(t-b)^{(\alpha)}$.

Definition 2.1 [18] Let $\alpha \geq 0$ and $f$ be a function defined on $[0, b]$. The fractional $q$-integral of the Riemann-Liouville type is defined by $\left(I_{q}^{0} f\right)(x)=f(x)$ and

$$
\left(I_{q}^{\alpha} f\right)(x)=\frac{1}{\Gamma_{q}(\alpha)} \int_{0}^{x}(x-q t)^{(\alpha-1)} f(t) d_{q} t, \quad \alpha>0, x \in[0, b] .
$$

Definition 2.2 [16] The fractional $q$-derivative of the Riemann-Liouville type of order $\alpha \geq 0$ is defined by $\left(D_{q}^{0} f\right)(x)=f(x)$ and

$$
\left(D_{q}^{\alpha} f\right)(x)=\left(D_{q}^{p} I_{q}^{p-\alpha} f\right)(x), \quad \alpha>0
$$

where $p$ is the smallest integer greater than or equal to $\alpha$.

Next, we list some properties about $q$-derivative and $q$-integral that are already known in the literature. 
Lemma $2.2[16,18]$ Let $\alpha, \beta \geq 0$ and $f$ be a function defined on $[0,1]$. Then the following formulas hold:

(i) $\left(I_{q}^{\beta} I_{q}^{\alpha} f\right)(x)=\left(I_{q}^{\alpha+\beta} f\right)(x)$;

(ii) $\left(D_{q}^{\alpha} I_{q}^{\alpha} f\right)(x)=f(x)$.

Lemma $2.3[18,20]$ Let $\alpha>0$ and $p$ be a positive integer. Then the following equality holds:

$$
\left(I_{q}^{\alpha} D_{q}^{p} f\right)(x)=\left(D_{q}^{p} I_{q}^{\alpha} f\right)(x)-\sum_{k=0}^{p-1} \frac{x^{\alpha-p+k}}{\Gamma_{q}(\alpha+k-p+1)}\left(D_{q}^{k} f\right)(0) .
$$

Lemma 2.4 [29] Let $X$ be a Banach space and $P \subseteq X$ be a cone. Suppose that $\Omega_{1}$ and $\Omega_{2}$ are bounded open sets contained in $X$ such that $0 \in \Omega_{1} \subseteq \bar{\Omega}_{1} \subseteq \Omega_{2}$. Suppose further that $S: P \cap\left(\bar{\Omega}_{2} \backslash \Omega_{1}\right) \rightarrow P$ is a completely continuous operator. If either

(i) $\|S u\| \leq\|u\|$ for $u \in P \cap \partial \Omega_{1}$ and $\|S u\| \geq\|u\|$ for $u \in P \cap \partial \Omega_{2}$, or

(ii) $\|S u\| \geq\|u\|$ for $u \in P \cap \partial \Omega_{1}$ and $\|S u\| \leq\|u\|$ for $u \in P \cap \partial \Omega_{2}$, then $S$ has at least one fixed point in $P \cap\left(\bar{\Omega}_{2} \backslash \Omega_{1}\right)$.

The next result is important in the sequel.

Lemma 2.5 Let $h \in C[0,1]$ be a given function. Then the boundary value problem

$$
\begin{aligned}
& \left(D_{q}^{\alpha} u\right)(t)+h(t)=0, \quad 0 \leq t \leq 1, \\
& D_{q}^{i} u(0)=0, \quad i=0,1, \ldots, n-2, \quad D_{q} u(1)=H_{1}(\varphi(u))+\int_{E} H_{2}(s, u(s)) d_{q} s,
\end{aligned}
$$

has a unique solution

$$
u(t)=\frac{t^{\alpha-1}}{[\alpha-1]_{q}}\left(H_{1}(\varphi(u))+\int_{E} H_{2}(s, u(s)) d_{q} s\right)+\int_{0}^{1} G(t, q s) h(s) d_{q} s,
$$

where

$$
G(t, q s)=\frac{1}{\Gamma_{q}(\alpha)} \begin{cases}(1-q s)^{(\alpha-2)} t^{\alpha-1}-(t-q s)^{(\alpha-1)}, & 0 \leq q s \leq t \leq 1 \\ (1-q s)^{(\alpha-2)} t^{\alpha-1}, & 0 \leq t \leq q s \leq 1\end{cases}
$$

Proof In view of Definition 2.2 and Lemma 2.2, we deduce

$$
I_{q}^{\alpha} D_{q}^{\alpha} u(t)=-I_{q}^{\alpha} h(t)
$$

and

$$
I_{q}^{\alpha} D_{q}^{n} I_{q}^{n-\alpha} u(t)=-I_{q}^{\alpha} h(t)
$$

It follows from Lemma 2.3,

$$
u(t)=c_{1} t^{\alpha-1}+c_{2} t^{\alpha-2}+\cdots+c_{n} t^{\alpha-n}-\frac{1}{\Gamma_{q}(\alpha)} \int_{0}^{t}(t-q s)^{(\alpha-1)} h(s) d_{q} s,
$$


where $c_{1}, \ldots, c_{n} \in \mathbb{R}$ are some constants to be determined. By the boundary condition $u(0)=0$, we get $c_{n}=0$. Now if $n \geq 2$, then differentiating both sides of (2.4) $j$ times for $j=1,2, \ldots, n-2$, we obtain

$$
\begin{aligned}
D_{q}^{j} u(t)= & {[\alpha-1]_{q} \cdots[\alpha-j]_{q} c_{1} t^{\alpha-j-1}+[\alpha-2]_{q} \cdots[\alpha-j-1]_{q} c_{2} t^{\alpha-j-2}+\cdots } \\
& +[\alpha+n-1]_{q} \cdots[\alpha-n-j+2]_{q} c_{n-1} t^{\alpha-n-j+1} \\
& -\frac{1}{\Gamma_{q}(\alpha)} \int_{0}^{t}[\alpha-1]_{q} \cdots[\alpha-j]_{q}(t-q s)^{(\alpha-j-1)} h(s) d_{q} s .
\end{aligned}
$$

From the boundary conditions $D_{q}^{i} u(0)=0$, for $i=1,2, \ldots, n-2$, it is easy to know $c_{2}=c_{3}=$ $\cdots=c_{n-1}=0$. Thus, (2.4) reduces to

$$
u(t)=c_{1} t^{\alpha-1}-\frac{1}{\Gamma_{q}(\alpha)} \int_{0}^{t}(t-q s)^{(\alpha-1)} h(s) d_{q} s .
$$

Differentiating both sides of (2.5), we obtain

$$
D_{q} u(1)=[\alpha-1]_{q} c_{1}-\frac{1}{\Gamma_{q}(\alpha)} \int_{0}^{1}[\alpha-1]_{q}(1-q s)^{(\alpha-2)} h(s) d_{q} s .
$$

Using the boundary condition $D_{q} u(1)=H_{1}(\varphi(u))+\int_{E} H_{2}(s, u(s)) d_{q} s$, we have

$$
c_{1}=\frac{1}{[\alpha-1]_{q}}\left(H_{1}(\varphi(u))+\int_{E} H_{2}(s, u(s)) d_{q} s\right)+\frac{1}{\Gamma_{q}(\alpha)} \int_{0}^{1}(1-q s)^{(\alpha-2)} h(s) d_{q} s .
$$

Hence,

$$
\begin{aligned}
u(t)= & \frac{t^{\alpha-1}}{[\alpha-1]_{q}}\left(H_{1}(\varphi(u))+\int_{E} H_{2}(s, u(s)) d_{q} s\right)+\frac{t^{\alpha-1}}{\Gamma_{q}(\alpha)} \int_{0}^{1}(1-q s)^{(\alpha-2)} h(s) d_{q} s \\
& -\frac{1}{\Gamma_{q}(\alpha)} \int_{0}^{t}(t-q s)^{(\alpha-1)} h(s) d_{q} s \\
= & \frac{t^{\alpha-1}}{[\alpha-1]_{q}}\left(H_{1}(\varphi(u))+\int_{E} H_{2}(s, u(s)) d_{q} s\right)+\int_{0}^{1} G(t, q s) h(s) d_{q} s .
\end{aligned}
$$

Remark 2.2 [20] Let $0<\tau<1$. Then $0<\tau^{\alpha-1}<1$ and $\min _{t \in[\tau, 1]} G(t, q s) \geq \tau^{\alpha-1} G(1, q s)$ for $s \in[0,1]$.

The following properties of the Green's function play important roles in this paper.

Lemma 2.6 [20] The function $G$ defined as (2.3) satisfies the following properties:

(1) $G(t, q s) \geq 0$ and $G(t, q s) \leq G(1, q s)$ for all $0 \leq s, t \leq 1$;

(2) $G(t, q s) \geq r(t) \max _{t \in[0,1]} G(t, q s)=r(t) G(1, q s)$ for all $0 \leq t, s \leq 1$ with $r(t)=t^{\alpha-1}$.

\section{Main results}

Let the Banach space $B=C[0,1]$ be endowed with the norm $\|u\|=\max _{t \in[0,1]}|u(t)|$. Let $\tau$ be a real constant with $0<\tau<1$ and define the cone $K \subset B$ by

$$
K=\left\{u \in C[0,1] \mid u(t) \geq 0, \min _{t \in[\tau, 1]} u(t) \geq r^{*}\|u\|, \varphi(u) \geq 0\right\},
$$

where $r^{*}=\min \left\{\frac{\min _{t \in[\tau, 1]} t^{\alpha-1}}{\max _{t \in[0,1]} t^{\alpha-1}}, \min _{t \in[\tau, 1]} r(t)\right\}=\min _{t \in[\tau, 1]} t^{\alpha-1}$, obviously, $r^{*} \in(0,1]$. 
Define the operator $F: K \rightarrow B$ by

$$
(F u)(t)=\frac{t^{\alpha-1}}{[\alpha-1]_{q}}\left(H_{1}(\varphi(u))+\int_{E} H_{2}(s, u(s)) d_{q} s\right)+\int_{0}^{1} G(t, q s) f(s, u(s)) d_{q} s .
$$

In order to get the integrated and rigorous theory, we make the following assumptions.

(H1) There are constants $C_{1}, C_{2}>0$ such that the functional $\varphi$ satisfies the inequality

$$
C_{2}\|u\| \leq \varphi(u) \leq C_{1}\|u\| \quad \text { for all } u \in C[0,1]
$$

(H2) For each given $\varepsilon>0$, there are $C_{3}>0$ and $G_{\varepsilon}>0$ such that

$$
\left|H_{1}(z)-C_{3} z\right|<\varepsilon C_{3} z, \quad \text { whenever } z>G_{\varepsilon} \text {. }
$$

(H3) There exists a function $T:[0,+\infty) \rightarrow[0,+\infty)$ satisfying the growth condition

$$
T(u) \leq C_{4} u
$$

for some $C_{4} \geq 0$. For each $\varepsilon>0$ given and $x \in[0,1]$, there is $M_{\varepsilon}>0$ such that

$$
\left|H_{2}(x, u)-T(u)\right|<\varepsilon T(u), \quad \text { whenever } u>M_{\varepsilon} \text {. }
$$

The following result plays an important role in the coming discussion.

Lemma 3.1 $F: K \rightarrow K$ is completely continuous.

Proof It is easy to see that the operator $F$ is continuous in view of the continuity of $G$ and $f$. By Lemmas 2.1 and 2.6, we have

$$
\begin{aligned}
& \min _{t \in[\tau, 1]} F u(t) \\
& \quad=\min _{t \in[\tau, 1]}\left[\frac{t^{\alpha-1}}{[\alpha-1]_{q}}\left(H_{1}(\varphi(u))+\int_{E} H_{2}(s, u(s)) d_{q} s\right)+\int_{0}^{1} G(t, q s) f(s, u(s)) d_{q} s\right] \\
& \quad=\min _{t \in[\tau, 1]}\left[\frac{t^{\alpha-1}}{[\alpha-1]_{q}}\left(H_{1}(\varphi(u))+\int_{E} H_{2}(s, u(s)) d_{q} s\right)\right]+\min _{t \in[\tau, 1]} \int_{0}^{1} G(t, q s) f(s, u(s)) d_{q} s \\
& \geq r_{0}\left[H_{1}(\varphi(u))+\int_{E} H_{2}(s, u(s)) d_{q} s\right]+\min _{t \in[\tau, 1]} \int_{0}^{1} r(t) \max _{t \in[0,1]} G(t, q s) f(s, u(s)) d_{q} s \\
& \quad \geq r_{0}\left[H_{1}(\varphi(u))+\int_{E} H_{2}(s, u(s)) d_{q} s\right]+\min _{t \in[\tau, 1]} r(t) \max _{t \in[0,1]} \int_{0}^{1} G(t, q s) f(s, u(s)) d_{q} s \\
& \geq r^{*}\|F u\|,
\end{aligned}
$$

where $r_{0}=\frac{\min _{t \in[\tau, 1]} t^{\alpha-1}}{\max _{t \in[0,1]} t^{\alpha-1}}$. Thus, $F(K) \subset K$.

Now let $\Omega \subset K$ be bounded, i.e., there exists a positive constant $M>0$ such that $\|u\| \leq M$ for all $u \in \Omega$. By the continuity of $H_{1}, H_{2}$, and $\varphi$, we easily see that $H_{1}$ and $H_{2}$ are bounded, 
so there exist constants $P>0$ and $Q>0$ such that $\left|H_{1}(\varphi(\Omega))\right| \leq P$ and $\left|H_{2}(t, u(t))\right| \leq Q$. Let $L=\max _{\|u\| \leq M}|f(t, u(t))|+1$. Then for $u \in \Omega$, from Lemma 2.6, we have

$$
\begin{aligned}
|F u(t)| & =\left|\frac{t^{\alpha-1}}{[\alpha-1]_{q}}\left(H_{1}(\varphi(u))+\int_{E} H_{2}(s, u(s)) d_{q} s\right)+\int_{0}^{1} G(t, q s) f(s, u(s)) d_{q} s\right| \\
& \leq\left|\frac{1}{[\alpha-1]_{q}}\left(H_{1}(\varphi(u))+\int_{E} H_{2}(s, u(s)) d_{q} s\right)+\int_{0}^{1} G(1, q s) f(s, u(s)) d_{q} s\right| \\
& \leq \frac{1}{[\alpha-1]_{q}}\left(\left|H_{1}(\varphi(u))\right|+\left|\int_{E} H_{2}(s, u(s)) d_{q} s\right|\right)+L \int_{0}^{1} G(1, q s) d_{q} s,
\end{aligned}
$$

we rearrange (3.3) as follows:

$$
\begin{aligned}
|F u(t)| & \leq \frac{1}{[\alpha-1]_{q}}\left(\left|H_{1}(\varphi(u))\right|+\left|\int_{E} H_{2}(s, u(s)) d_{q} s\right|\right)+L \int_{0}^{1} G(1, q s) d_{q} s \\
& \leq \frac{1}{[\alpha-1]_{q}}\left(\left|H_{1}(\varphi(u))\right|+\int_{E}\left|H_{2}(s, u(s))\right| d_{q} s\right)+L \int_{0}^{1} G(1, q s) d_{q} s \\
& \leq \frac{1}{[\alpha-1]_{q}}(P+Q m(E))+L \int_{0}^{1} G(1, q s) d_{q} s .
\end{aligned}
$$

Hence, $F(\Omega)$ is bounded.

On the other hand, for any given $\varepsilon>0$, there exists $\delta>0$ small enough, such that $\mid F u\left(t_{2}\right)-$ $F u\left(t_{1}\right) \mid<\varepsilon$ holds for each $u \in \Omega$ and $0 \leq t_{1} \leq t_{2} \leq 1$ with $\left|t_{2}-t_{1}\right|<\delta$, that is to say, $F(\Omega)$ is equicontinuous. In fact,

$$
\begin{aligned}
& \left|F u\left(t_{2}\right)-F u\left(t_{1}\right)\right| \\
& \leq\left|t_{2}^{\alpha-1}-t_{1}^{\alpha-1}\right| \mid\left[\frac{1}{[\alpha-1]_{q}}\left(H_{1}(\varphi(u))+\int_{E} H_{2}(s, u(s)) d_{q} s\right)\right. \\
& \left.\quad+\int_{0}^{1} \frac{(1-q s)^{(\alpha-1)}}{\Gamma_{q}(\alpha)} f(s, u(s)) d_{q} s\right] \mid \\
& \quad+\frac{1}{\Gamma_{q}(\alpha)}\left|\int_{0}^{t_{2}}\left(t_{2}-q s\right)^{(\alpha-2)} f(s, u(s)) d_{q} s-\int_{0}^{t_{1}}\left(t_{1}-q s\right)^{(\alpha-2)} f(s, u(s)) d_{q} s\right| \\
& \leq\left|t_{2}^{\alpha-1}-t_{1}^{\alpha-1}\right|\left[\frac{1}{[\alpha-1]_{q}}\left(H_{1}(\varphi(u))+\int_{E} H_{2}(s, u(s)) d_{q} s\right)\right. \\
& \left.\quad+\int_{0}^{1} \frac{(1-q s)^{(\alpha-1)}}{\Gamma_{q}(\alpha)}|f(s, u(s))| d_{q} s\right] \\
& \quad+\frac{1}{\Gamma_{q}(\alpha)}\left|\int_{0}^{1} t_{2}^{\alpha-2}(1-q s) f(s, u(s)) d_{q} s-\frac{1}{\Gamma_{q}(\alpha)} \int_{0}^{1} t_{1}^{\alpha-2}(1-q s) f(s, u(s)) d_{q} s\right| \\
& \leq\left|t_{2}^{\alpha-1}-t_{1}^{\alpha-1}\right|\left[\frac{1}{[\alpha-1]_{q}}(P+Q m(E))+L \int_{0}^{1} \frac{(1-q s)^{(\alpha-1)}}{\Gamma_{q}(\alpha)} d_{q} s\right] \\
& \quad+\left|t_{2}^{\alpha-2}-t_{1}^{\alpha-2}\right| \frac{L}{\Gamma_{q}(\alpha)} \int_{0}^{1}(1-q s) d_{q} s .
\end{aligned}
$$

Now, we estimate $t_{2}^{\alpha-1}-t_{1}^{\alpha-1}$ (we discuss $t_{2}^{\alpha-2}-t_{1}^{\alpha-2}$ in the same way, the proof here is omitted): 
(1) for $0 \leq t_{1}<\delta, \delta \leq t_{2}<2 \delta, t_{2}^{\alpha-1}-t_{1}^{\alpha-1} \leq t_{2}^{\alpha-1}<(2 \delta)^{\alpha-1} \leq 2 \delta$;

(2) for $0 \leq t_{1}<t_{2} \leq \delta, t_{2}^{\alpha-1}-t_{1}^{\alpha-1} \leq t_{2}^{\alpha-1}<\delta^{\alpha-1} \leq 2 \delta$;

(3) for $\delta \leq t_{1}<t_{2} \leq 1$, from the mean value theorem of differentiation, we have $t_{2}^{\alpha-1}-t_{1}^{\alpha-1} \leq(\alpha-1)\left(t_{2}-t_{1}\right) \leq 2 \delta$.

Thus, we have

$$
\left|F u\left(t_{2}\right)-F u\left(t_{1}\right)\right|<\varepsilon \text {. }
$$

By means of the Arzela-Ascoli theorem, $F: K \rightarrow K$ is completely continuous. The proof is complete.

Theorem 3.1 Assume that the nonlinearity $f(t, u)$ splits in the sense that $f(t, u)=a(t) g(u)$, for continuous functions $a:[0,1] \rightarrow[0,+\infty)$ and $g:[0, \infty) \rightarrow[0,+\infty)$ such that $\lim _{u \rightarrow 0^{+}} \frac{g(u)}{u}=+\infty$ and $\lim _{u \rightarrow+\infty} \frac{g(u)}{u}=0$. Suppose conditions (H1)-(H3) and

$$
C_{1} C_{3}+C_{4} m(E)<1
$$

hold, where $E \subseteq(0,1]$ is a closed subinterval. Then the boundary value problem (1.1)-(1.2) has at least one solution.

Proof Begin by selecting the number $\eta_{1}$ such that

$$
\eta_{1} \int_{0}^{1} \gamma^{*} G(1, q s) a(s) d_{q} s>1 .
$$

Now, there exists a number $r_{1}>0$ such that $g(u) \geq \eta_{1} u$ for $0<u<r_{1}$. Then take the open set

$$
\Omega_{r_{1}}=\left\{u \in B:\|u\|<r_{1}\right\} .
$$

By Remark 2.2, Lemma 2.6, and (3.6), for $u \in K \cap \partial \Omega_{r_{1}}$, we find

$$
\begin{aligned}
(F u)(t) & =\frac{t^{\alpha-1}}{[\alpha-1]_{q}}\left(H_{1}(\varphi(u))+\int_{E} H(s, u(s)) d_{q} s\right)+\int_{0}^{1} G(t, q s) f(s, u(s)) d_{q} s \\
& \geq \int_{0}^{1} G(t, q s) a(s) g(u(s)) d_{q} s \\
& \geq \int_{0}^{1} G(t, q s) a(s) \eta_{1} u(s) d_{q} s \\
& \geq\|u\| \eta_{1} \int_{0}^{1} \gamma^{*} G(1, q s) a(s) d_{q} s \\
& \geq\|u\| .
\end{aligned}
$$

Hence, $\|F u\| \geq\|u\|$, that is, $F$ is a cone expansion on $K \cap \partial \Omega_{r_{1}}$.

On the other hand, we consider two cases:

Case 1 . Suppose that $g$ is bounded for $u \in[0,+\infty)$. We may find $r_{2}>0$ sufficiently large such that

$$
g(u) \leq r_{2} \quad \text { for all } u \geq 0 .
$$


Condition (3.5) implies the existence of $\varepsilon>0$ such that

$$
C_{1} C_{3} \varepsilon+C_{1} C_{3}+\varepsilon+\left(C_{4}+C_{4} \varepsilon\right) m(E) \leq 1 .
$$

Now if $\|u\| \geq \frac{G_{\varepsilon}}{C_{2}}$, then by (H1) we get $\varphi(u) \geq G_{\varepsilon}$. According to condition (H2), it follows that

$$
\left|H_{1}(\varphi(u))-C_{3} \varphi(u)\right|<\varepsilon C_{3} \varphi(u)
$$

for all $u \in C[0,1]$ with $\|u\| \geq \frac{G_{\varepsilon}}{C_{2}}$.

Next, since $E \subseteq(0,1]$ is a closed subinterval, we may select $0<\tau<1$ such that $E \subseteq[\tau, 1]$. Then for each $u \in K$,

$$
\min _{t \in E} u(t) \geq \min _{t \in[\tau, 1]} u(t) \geq \gamma^{*}\|u\|
$$

Thus combining condition $(\mathrm{H} 3)$ we see that

$$
\left|H_{2}(s, u(s))-T(u(s))\right|<\varepsilon T(u(s)) \quad \text { for each } s \in E \text { with }\|u\| \geq \frac{M_{\varepsilon}}{\gamma^{*}} .
$$

Take $r_{2}^{*}=\max \left\{\frac{2 r_{1}}{\gamma^{*}}, r_{2}, \frac{G_{\varepsilon}}{C_{2}}, \frac{M_{\varepsilon}}{\gamma^{*}}\right\}$. Set $\Omega_{r_{2}^{*}}=\left\{u \in B:\|u\|<r_{2}^{*}\right\}$.

From (3.8) we may assume without loss of generality that

$$
g(u) \leq \frac{r_{2}}{\int_{0}^{1} G(1, q s) a(s) d_{q} s} \varepsilon .
$$

Then by (3.9), (3.10), (3.12), and (3.13), for each $u \in K \cap \partial \Omega_{r_{2}^{*}}$, we have

$$
\begin{aligned}
\|F u\| \leq & H_{1}(\varphi(u))+\int_{E} H_{2}(s, u(s)) d_{q} s+\int_{0}^{1} G(t, q s) f(s, u(s)) d_{q} s \\
\leq & H_{1}(\varphi(u))+\int_{E} H_{2}(s, u(s)) d_{q} s+\int_{0}^{1} G(1, q s) a(s) g(u(s)) d_{q} s \\
\leq & \left|H_{1}(\varphi(u))-C_{3} \varphi(u)\right|+C_{3}|\varphi(u)|+\int_{E}\left|H_{2}(s, u(s))-T(u(s))\right| d_{q} s \\
& +\int_{E}|T(u(s))| d_{q} s+\int_{0}^{1} r_{2} \varepsilon d_{q} s \\
\leq & \varepsilon C_{1} C_{3}\|u\|+C_{1} C_{3}\|u\|+m(E) C_{4}(1+\varepsilon)\|u\|+\varepsilon\|u\| \\
\leq & {\left[C_{1} C_{3} \varepsilon+\varepsilon+C_{1} C_{3}+m(E) C_{4}(1+\varepsilon)\right]\|u\| } \\
\leq & \|u\| .
\end{aligned}
$$

Case 2. Suppose that $g$ is unbounded at $+\infty$. By condition $\lim _{u \rightarrow+\infty} \frac{g(u)}{u}=0$, there exists a number $r_{3}>0$ such that for $u>r_{4}$ we find that $g(u) \leq \eta_{2} u$, where $\eta_{2}$ meets

$$
\eta_{2} \int_{0}^{1} G(1, q s) a(s) d_{q} s \leq \varepsilon
$$


Noting that $g$ is unbounded at $+\infty$, we may find $r_{3}^{*}>\max \left\{\frac{2 r_{1}}{\gamma^{*}}, r_{3}, \frac{G_{\varepsilon}}{C_{2}}, \frac{M_{\varepsilon}}{\gamma^{*}}\right\}$ such that

$$
g(u) \leq g\left(r_{3}\right) \text { for } 0<u \leq r_{3}^{*}
$$

Now, put $\Omega_{r_{2}^{*}}=\left\{u \in B:\|u\|<r_{3}^{*}\right\}$. Then for each $u \in K \cap \partial \Omega_{r_{3}^{*}}$ we find that by (3.9), (3.10), (3.12), (3.15), and (3.16),

$$
\begin{aligned}
\|F u\| \leq & H_{1}(\varphi(u))+\int_{E} H_{2}(s, u(s)) d_{q} s+\int_{0}^{1} G(t, q s) f(s, u(s)) d_{q} s \\
\leq & H_{1}(\varphi(u))+\int_{E} H_{2}(s, u(s)) d_{q} s+\int_{0}^{1} G(1, q s) a(s) g(u(s)) d_{q} s \\
\leq & \left|H_{1}(\varphi(u))-C_{3} \varphi(u)\right|+C_{3}|\varphi(u)|+\int_{E}\left|H_{2}(s, u(s))-T(u(s))\right| d_{q} s \\
& +\int_{E}|T(u(s))| d_{q} s+\int_{0}^{1} G(1, q s) a(s) g\left(r_{3}^{*}\right) d_{q} s \\
\leq & C_{3} \varepsilon \varphi(u)+C_{1} C_{3}\|u\|+\int_{E}(1+\varepsilon) T(u(s)) d_{q} s \\
& +\int_{0}^{1} G(1, q s) a(s) \eta_{2}\|u\| d_{q} s \\
\leq & \varepsilon C_{1} C_{3}\|u\|+C_{1} C_{3}\|u\|+m(E) C_{4}(1+\varepsilon)\|u\|+\varepsilon\|u\| \\
\leq & {\left[C_{1} C_{3} \varepsilon+\varepsilon+C_{1} C_{3}+m(E) C_{4}(1+\varepsilon)\right]\|u\| } \\
\leq & \|u\| .
\end{aligned}
$$

Hence, $\|F u\| \leq\|u\|$.

To summarize, we conclude from (3.14) and (3.17) that $F$ is a cone compression on $K \cap$ $\partial \Omega_{r_{2}^{*}}$.

With the help of Lemma 2.4 we can now deduce the existence of function $u_{0} \in K \cap \bar{\Omega}_{r_{2}^{*}} \backslash$ $\Omega_{r_{1}}$ such that $F u_{0}=u_{0}$. Hence, the problem (1.1)-(1.2) has at least one solution. The proof is completed.

Next, by choosing suitable forms of $H_{1}$ and $\varphi$, we present the corresponding boundary value problems with separated boundary conditions, integral boundary conditions and multi-point boundary conditions as corollaries of Theorem 3.1 to illustrate the universality and generalization of our results.

Corollary 3.1 Assume $f(t, u)$ is defined as in Theorem 3.1 and $(\mathrm{H} 1)-(\mathrm{H} 3)$ hold. If $n=3$, $H_{1}(\varphi(u))+\int_{E} H_{2}(s, u(s)) d_{q} s=\beta \geq 0$. Then the boundary value problem

$$
\begin{aligned}
& \left(D_{q}^{\alpha} u\right)(t)+f(t, u(t))=0, \quad 0<t<1,2<\alpha \leq 3, \\
& u(0)=D_{q} u(0)=0, \quad D_{q} u(1)=\beta,
\end{aligned}
$$

has at least one solution.

This existence result of positive solution for the boundary value problem (3.18)-(3.19) has been studied by Ferreira in [20] and Yang et al. in [27]. 
Corollary 3.2 Assume $f(t, u)$ is defined as in Theorem 3.1, $\varphi(u)=\int_{T} u(s) d_{q}$ s. Then $\varphi$ is linear functional, $T=[a, b] \subseteq(0,1]$ is a closed subinterval, just need $m(T) \leq C_{1}$, where $m$ is Lebesgue measure. If (H1)-(H3) hold, in addition

$$
C_{1} C_{3}+C_{4} m(E)<1
$$

then the boundary value problem (3.20)-(3.21) with integral boundary conditions

$$
\begin{aligned}
& \left(D_{q}^{\alpha} u\right)(t)+f(t, u(t))=0, \quad 0<t<1, n-1<\alpha \leq n, \\
& u(0)=D_{q} u(0)=0, \quad D_{q} u(1)=H_{1}\left(\int_{T} u(s) d_{q} s\right)+\int_{E} H_{2}(s, u(s)) d_{q} s,
\end{aligned}
$$

has at least one solution.

Corollary 3.3 Assume $f(t, u)$ is defined as in Theorem 3.1, $\varphi(u)=\sum_{i=1}^{m} a_{i} u\left(\xi_{i}\right)$. Then $\varphi$ is linear functional, just need $\sum_{i=1}^{m}\left|a_{i}\right| \leq C_{1}, E \subseteq(0,1]$ is a closed subinterval. If (H1)-(H3) hold, moreover,

$$
C_{1} C_{3}+C_{4} m(E)<1
$$

then the boundary value problem (3.22)-(3.23) with multi-point boundary conditions

$$
\begin{aligned}
& \left(D_{q}^{\alpha} u\right)(t)+f(t, u(t))=0, \quad 0<t<1, n-1<\alpha \leq n, \\
& u(0)=D_{q} u(0)=0, \quad D_{q} u(1)=H_{1}\left(\sum_{i=1}^{m} a_{i} u\left(\xi_{i}\right)\right)+\int_{E} H_{2}(s, u(s)) d_{q} s,
\end{aligned}
$$

has at least one solution.

\section{Example}

In this section, we will give an example to expound our main results.

Example 4.1 Consider the following boundary value problem:

$$
\begin{aligned}
& \left(D_{q}^{\alpha} u\right)(t)+f(t, u)=0, \quad 0<t<1, \\
& u(0)=D_{q} u(0)=0, \quad D_{q} u(1)=\sum_{j=1}^{m} 2 a_{j} \xi_{j}+e^{-\sum_{j=1}^{m} 2 a_{j} \xi_{j}}+\int_{E} u(s) d_{q} s,
\end{aligned}
$$

here $2<\alpha \leq 3, a_{j} \geq 0$ with $\frac{1}{4} \leq \sum_{j=1}^{m} a_{j} \leq \frac{3}{4}$ and $\xi_{j} \in\left[\frac{1}{100}, \frac{1}{10}\right]$ for $j=1,2, \ldots, m, E \subseteq(0,1]$ is a closed subinterval, $\varphi(u)=\sum_{j=1}^{m} 2 a_{j} \xi_{j}, H_{1}(\varphi(u))=\varphi(u)+e^{-\varphi(u)}$ and $\int_{E} H_{2}(s, u(s)) d_{q} s=$ $\int_{E} u(s) d_{q} s$ satisfies $(\mathrm{H} 3)$.

Since $a_{j} \geq 0$ and $\xi_{j} \in\left[\frac{1}{100}, \frac{1}{10}\right]$, then $|\varphi(u)|=\sum_{j=1}^{m} 2 a_{j} \xi_{j}$. Choosing $C_{1}=\frac{3}{20}$ and $C_{2}=\frac{1}{40}$, we have

$$
\frac{1}{200} \leq|\varphi(u)|=\sum_{j=1}^{m} 2 a_{j} \xi_{j} \leq \frac{3}{20} .
$$


For each $\varepsilon>0$, setting $C_{3}=1$, it is clear that

$$
\lim _{\varphi(u) \rightarrow \infty}\left|H_{1}(\varphi(u))-\varphi(u)\right|=\left|\varphi(u)+e^{-\varphi(u)}-\varphi(u)\right|=0,
$$

so (H2) holds.

If only the given function $f(t, u)$ satisfies $f(t, u)=a(t) g(u)$, for continuous functions $a$ : $[0,1] \rightarrow[0,+\infty)$ and $g: \mathbb{R} \rightarrow[0,+\infty)$ such that $\lim _{u \rightarrow 0^{+}} \frac{g(u)}{u}=+\infty$ and $\lim _{u \rightarrow+\infty} \frac{g(u)}{u}=0$, in addition,

$$
C_{1} C_{3}+C_{4} m(E)<1
$$

then, by Theorem 3.1, the boundary value problem (4.1)-(4.2) has at least one solution.

\section{Competing interests}

The authors declare that they have no competing interests.

\section{Authors' contributions}

The authors declare that the study was realized in collaboration with the same responsibility. All authors read and approved the final manuscript.

\section{Author details}

'School of Mathematical Sciences, University of Jinan, Jinan, Shandong 250022, P.R. China. ${ }^{2}$ School of Control Science and Engineering, University of Jinan, Jinan, Shandong 250022, P.R. China.

\section{Acknowledgements}

The authors sincerely thank the reviewers for their valuable suggestions and useful comments, which have led to the present improved version of the original manuscript. This research is supported by the Natural Science Foundation of China (61374074), Shandong Provincial Natural Science Foundation (ZR2012AM009, ZR2013AL003), also supported by Graduate Innovation Foundation of University of Jinan (YCX13013).

Received: 10 September 2014 Accepted: 9 December 2014 Published: 19 Dec 2014

\section{References}

1. Miller, K, Ross, B: An Introduction to the Fractional Calculus and Fractional Differential Equations. A Wiley-Interscience Publication. Wiley, New York (1993)

2. Oldham, K, Spanier, J: The Fractional Calculus. Academic Press, New York (1974)

3. Magin, R: Fractional Calculus in Bioengineering. Begell House, Redding (2006)

4. Zhao, Y, Sun, S, Han, Z, Li, Q: The existence of multiple positive solutions for boundary value problems of nonlinear fractional differential equations. Commun. Nonlinear Sci. Numer. Simul. 16(4), 2086-2097 (2011)

5. Zhao, Y, Sun, S, Han, Z, Zhang, M: Positive solutions for boundary value problems of nonlinear fractional differential equations. Appl. Math. Comput. 217, 6950-6958 (2011)

6. Feng, W, Sun, S, Han, Z, Zhao, Y: Existence of solutions for a singular system of nonlinear fractional differential equations. Comput. Math. Appl. 62(3), 1370-1378 (2011)

7. Goodrich, C: On discrete sequential fractional boundary value problems. J. Math. Anal. Appl. 385(1), 111-124 (2012)

8. Atici, F, Sengül, S: Modeling with fractional difference equations. J. Math. Anal. Appl. 369, 1-9 (2010)

9. Jackson, F: On q-functions and a certain difference operator. Trans. R. Soc. Edinb. 46, 253-281 (1908)

10. Jackson, F: On q-definite integrals. Q. J. Pure Appl. Math. 41, 193-203 (1910)

11. Kac, V, Cheung, P: Quantum Calculus. Springer, New York (2002)

12. Abdel-Gawad, H, Aldailami, A: On q-dynamic equations modelling and complexity. Appl. Math. Model. 34(3), 697-709 (2010)

13. Field, C, Joshi, N, Nijhoff, F: q-Difference equations of KdV type and Chazy-type second-degree difference equations. J. Phys. A, Math. Theor. 41(33), 1-13 (2008)

14. Abreu, L: Sampling theory associated with q-difference equations of the Sturm-Liouville type. J. Phys. A 38(48), 10311-10319 (2005)

15. Rajković, P, Marinković, S, Stanković, M: On q-analogues of Caputo derivative and Mittag-Leffler function. Fract. Calc. Appl. Anal. 4(10), 359-373 (2007)

16. Rajković, P, Marinković, S, Stanković, M: Fractional integrals and derivatives in q-calculus. Appl. Anal. Discrete Math. 1(1), 311-323 (2007)

17. Al-Salam, W: Some fractional q-integrals and q-derivatives. Proc. Edinb. Math. Soc. 15(2), 135-140 (1966/1967)

18. Agarwal, RP: Certain fractional $q$-integrals and q-derivatives. Proc. Camb. Philos. Soc. 66, 365-370 (1969)

19. Annaby, M, Mansour, Z: q-Fractional Calculus and Equations. Springer, New York (2012)

20. Ferreira, R: Positive solutions for a class of boundary value problems with fractional $q$-differences. Comput. Math. Appl. 61, 367-373 (2011) 
21. Liang, S, Zhang, J: Existence and uniqueness of positive solutions for three-point boundary value problem with fractional q-differences. J. Appl. Math. Comput. 40, 277-288 (2012)

22. Zhou, W, Liu, H: Existence solutions for boundary value problem of nonlinear fractional q-difference equations. Adv. Differ. Equ. 2013, 113 (2013)

23. Agarwal, RP, Ahmad, B, Alsaedi, A, Al-Hutami, H: On nonlinear fractional q-difference equations involving two fractional orders with three-point nonlocal boundary conditions. Dyn. Contin. Discrete Impuls. Syst., Ser. A Math. Anal. 21, 135-151 (2014)

24. Ahmad, B, Nieto, JJ, Alsaedi, A, Al-Hutami, H: Existence of solutions for nonlinear fractional q-difference integral equations with two fractional orders and nonlocal four-point boundary conditions. J. Franklin Inst. 351, $2890-2909$ (2014)

25. Ahmad, B, Ntouyas, SK: Existence of solutions for nonlinear fractional q-difference inclusions with nonlocal Robin (separated) conditions. Mediterr. J. Math. 10(3), 1333-1351 (2013)

26. Ahmad, B, Ntouyas, SK, Purnaras, I: Existence results for nonlocal boundary value problems of nonlinear fractional q-difference equations. Adv. Differ. Equ. 2012, 140 (2012). doi:10.1186/1687-1847-2012-140

27. Yang, L, Chen, $H$, Luo, Z: Successive iteration and positive solutions for boundary value problem of nonlinear fractional q-difference equation. J. Appl. Math. Comput. 42, 89-102 (2013)

28. Goodrich, C: On a nonlocal BVP with nonlinear boundary conditions. Results Math. 63, 1351-1364 (2013)

29. Krasnoselskii, M: Positive Solutions of Operator Equations. Noordhoff, Groningen (1964)

10.1186/1687-1847-2014-326

Cite this article as: Li et al.: Existence of solutions for fractional $q$-difference equation with mixed nonlinear boundary conditions. Advances in Difference Equations 2014, 2014:326

\section{Submit your manuscript to a SpringerOpen ${ }^{\circ}$ journal and benefit from:}

- Convenient online submission

- Rigorous peer review

- Immediate publication on acceptance

Open access: articles freely available online

- High visibility within the field

- Retaining the copyright to your article 